\title{
Dynamics of nonlinear oscillators with time-varying conjugate coupling
}

\author{
KIRAN YADAV $^{1}$, AMIT SHARMA $^{1,2}$ and MANISH DEV SHRIMALI ${ }^{1, *}$ \\ ${ }^{1}$ Department of Physics, Central University of Rajasthan, Ajmer 305 817, India \\ ${ }^{2}$ The Institute of Mathematical Science, CIT Campus, Taramani, Chennai 600 113, India \\ *Corresponding author. E-mail: shrimali@curaj.ac.in
}

\begin{abstract}
We explore the dynamical consequences of time-varying conjugate coupling in a system of nonlinear oscillators. We analyze the behavior of coupled systems with respect to the coupling switching frequency using normalized average synchronization error and average amplitude. We show that this form of time-varying interaction can induce an anomalous transition like the emergence of oscillation as well as the intermittent state with different dynamical states. This behavior is analyzed by numerical studies of specific cases of the Rössler oscillator and an ecological model of predator-prey systems.
\end{abstract}

Keywords. Conjugate coupling; time varying coupling.

\section{PACS Nos 05.45.Xt}

\section{Introduction}

The interaction of nonlinear systems typically leads to a variety of significant behaviors, one of the most intriguing of which is synchronization, that is, the coordination of a particular dynamical property of their motion $[1,2]$. A variety of synchrony behavior like complete synchronization, phase synchronization, lag synchronization, partial synchronization, and relay synchronization are achieved by coupling or external driving (deterministic and stochastic) in the coupled systems, which depend on the nature of coupling (forcing) such as time delay or nonlinear coupling (forcing) as well as strength or intensity of interaction (force) [2-5]. The particular type of interaction between dissimilar (conjugate) variables of dynamical systems induces synchronization $[6,7]$ as well as amplitude death [4] in the absence of time delay. This type of interaction or coupling also seems to be capable of generating rhythms in the coupled systems, which exhibit fixed-point behavior [10]. Typical examples of such interaction between conjugate variables are coupled semiconductor lasers, epidemiology, ecosystem, electrical circuits, and many more $[8,9,11]$.

In all these existing studies, the interaction or coupling among the systems is constant over time. However, the strength or form of interaction between systems can vary with time [12-20]. In such cases, it is important to study the global behavior of the coupled systems which depends on the interplay between the variable strength of dynamic interaction and switching frequency between dynamic interaction strengths. The rate of variation in coupling may be stochastic or periodic [13]. It was explored in an experimental work on synchronization of two coupled Chua's circuits [14]. In biological systems, the possibility of time-varying interaction has been studied, where it is stated that molecular networks can undergo systematic rewiring at each time step [12]. Another type of time-varying interaction in coupled oscillators is studied in Ref. [15], where each individual oscillator always has time-independent linear self-feedback while its interaction with other oscillators is modulated by a time-varying nonlinear function. In another study, a time-dependent on-off coupling is introduced in a network of regular lattice of cells [13]. The stability of synchronized state in time-varying networks is also explored by Kohar et al. [16]. Suppression and revival of oscillations were observed by switching the coupling form in a system of coupled oscillators [17]. Moreover, the study of the spread of epidemics in timevarying networks leads to the result that if the network changes more rapidly, the disease cycle becomes more synchronized which denotes the beginning of epidemics in the system [18].

Recently, the synchronization properties of a network consisting of heterogeneous oscillators were studied with a time-varying coupling [19], which shows that switching networks exhibit behavior similar to a static network if the switching of coupling or node is fast enough. The behavior of such a network is studied by time average of the switching connectivity. Such kind 
of dynamic coupling was also studied in a system of two Rössler oscillators where the coupling strength switched between two constant values with a given frequency [20]. Here, synchronization was observed by switching coupling between two values which fall outside the synchronization region. Considering the effect of switching coupling between similar variables, it is obvious and interesting to ask how the dynamics of oscillators will change when interaction via dissimilar (conjugate) variables changes with time as there are many ecological and other systems having dynamic interaction via conjugate variables, e.g., cross-predation, etc. In this work, we study the effect of switching coupling on nonlinear oscillators that interact via conjugate variables. We study Rössler as well as Truscott-Brindley oscillators interacting through timevarying conjugate coupling which switches periodically between two fixed values with a given frequency [20]. The switching frequency is taken as a parameter to study the dynamical behavior of the system where frequency plays an important role in the collective behaviour of coupled system leading to various dynamical states including in-phase and anti-phase synchronization as well as suppression and revival of oscillations.

\section{The model system and results}

We first consider two identical Rössler systems coupled via conjugate variables with coupling strength $k$. The equations governing the two coupled systems are written as

$$
\begin{aligned}
& \dot{x}_{1,2}=-y_{1,2}-z_{1,2}+k\left(y_{2,1}-x_{1,2}\right) \\
& \dot{y}_{1,2}=x_{1,2}+a y_{1,2} \\
& \dot{z}_{1,2}=b+z_{1,2}\left(x_{1,2}-c\right)
\end{aligned}
$$

where the parameters are $a=0.2, b=0.2$ and $c=7$. Such coupling scheme is studied in Ref. [6] where synchronization in coupled oscillators is observed. Conjugate coupling induces the amplitude death as well as the oscillation death in coupled oscillators [4].

To study the effect of conjugate coupling, we use two order parameters. One of the order parameter is the normalized average synchronization error $E$, to ensure that all the variables of the coupled systems are synchronized [20], which is calculated as

$E=\frac{1}{M} \sum_{h=1}^{M} \frac{\sqrt{\left(x_{1}(h)-x_{2}(h)\right)^{2}+\left(y_{1}(h)-y_{2}(h)\right)^{2}+\left(z_{1}(h)-z_{2}(h)\right)^{2}}}{\sqrt{x_{1}(h)^{2}+y_{1}(h)^{2}+z_{1}(h)^{2}+x_{2}(h)^{2}+y_{2}(h)^{2}+z_{2}(h)^{2}}}$.

For the case of complete synchronization $E=0$, otherwise, it is positive. The second order parameter is the average amplitude $A$ of the oscillation of the coupled system. It is calculated numerically using the difference between the global maximum and minimum of the time series of the oscillators over a sufficiently long time interval and then averaged over all oscillators at a particular parameter value [21]. The average amplitude $A$ is defined as

$A=\frac{1}{N} \sum_{i=1}^{N}\left[\left\langle x_{i, \max }\right\rangle_{t}-\left\langle x_{i, \min }\right\rangle_{t}\right]$

where $N$ is the number of coupled systems. The average amplitude $A=0$ when the coupled systems are in the stationary state, and for an oscillatory state $A>0$. The dynamical equations are solved using a fourth-order Runge-Kutta scheme with time-step $d t=0.01$.

The normalized average synchronization error $E$ and average amplitude $A$ as a function of coupling strength $k$ for the Rössler oscillators with conjugate coupling are shown in figure 1 . As the coupling strength is varied, the oscillators are synchronized in the range $k=0.13$ to 0.19 . A window of unsynchronized motion is observed around $k \sim 0.19$. With further increase of the coupling strength, the coupled system goes to the steady state through reverse Hopf bifurcation at $k=0.2$ [4]. Therefore, we observed three dynamical regimes, no-synchronization state (NS), complete synchronization state (CS), and amplitude death (AD) in conjugate-coupled nonlinear oscillators.

Now, we consider two identical Rössler oscillators coupled via a time-varying conjugate coupling. The dynamical equations in this case are

$$
\begin{aligned}
& \dot{x}_{1,2}=-y_{1,2}-z_{1,2}+k(t)\left(y_{2,1}-x_{1,2}\right) \\
& \dot{y}_{1,2}=x_{1,2}+a y_{1,2} \\
& \dot{z}_{1,2}=b+z_{1,2}\left(x_{1,2}-c\right)
\end{aligned}
$$




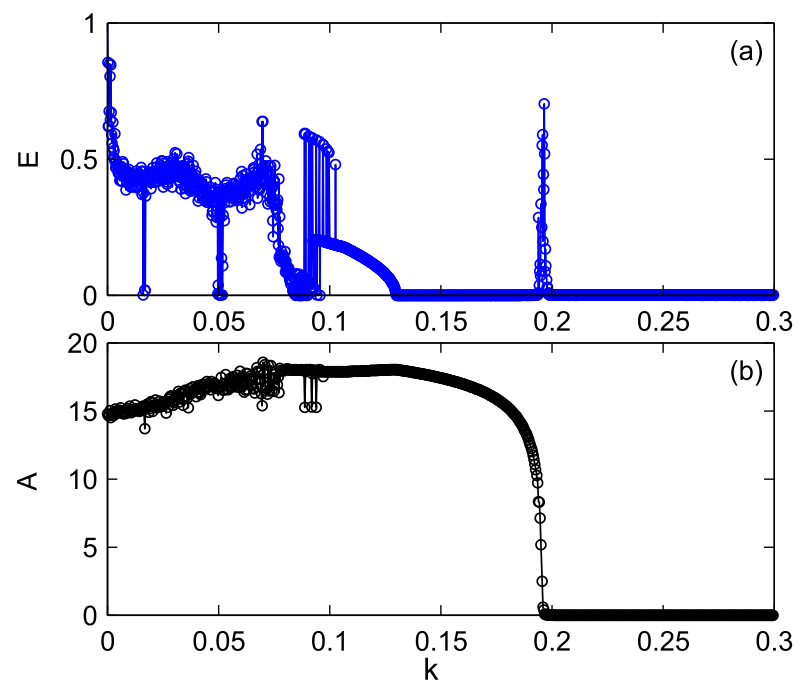

Figure 1. (a) Normalized average synchronization error $E$ and (b) average amplitude $A$ as a function of coupling strength $k$ for conjugate-coupled Rössler oscillators with static coupling.

The coupling strength $k(t)$ is a function of time and it is given by $k(t)=k_{1}+\frac{k_{2}-k_{1}}{2}(\operatorname{sgn}(\cos (\omega t))+1)$, where $\operatorname{sgn}(x)=1$ if $x>0$ and $\operatorname{sgn}(x)=-1$ otherwise. The effective coupling $k(t)$ switches between two constant values of coupling strength $k_{1}$ and $k_{2}$ with a frequency $\omega$.

To study the effect of the time-varying interaction in such coupled Rössler oscillators, we choose the coupling strength $k_{1}$ and $k_{2}$ from two different dynamical regimes. Now, based on the choice of $k_{1}$ and $k_{2}$, we consider two cases, (1) $\mathrm{C} 1$ : both $k_{1}$ and $k_{2}$ from no-synchronization regimes, and (2) $\mathrm{C} 2: k_{1}$ and $k_{2}$ from synchronization and amplitude death regimes respectively.

First, we study the $\mathrm{C} 1$ case and choose both coupling strength values $k_{1}=0.06$ and $k_{2}=0.196$ from the no-synchronization regime in figure 1 . We have plotted the average synchronization error $E$ and the average amplitude $A$ as a function of switching frequency $\omega$ in figure 2 . Here, some windows of synchronized motion along with no-synchronization are obtained. For low switching frequency, coupled Rössler oscillators are in no-synchronization regime till $\omega=0.5$, while for higher frequency, we obtain the synchronization in the range of $\omega=0.6$ to 0.85 and $\omega=1.15$ to 1.4 as shown in figure 2a. For higher switching frequency around 1.4 to 2.5 , coupled systems show multiple transitions between synchronized and unsynchronized states. In this case, we do not observe the death regime in the coupled system as shown in figure $2 b$.

For $\mathrm{C} 2$ case, we choose the coupling from two different dynamical regimes at $k_{1}=0.15(\mathrm{SS})$ and $k_{2}=0.3$ (AD). $E$ and $A$ are plotted against switching frequency

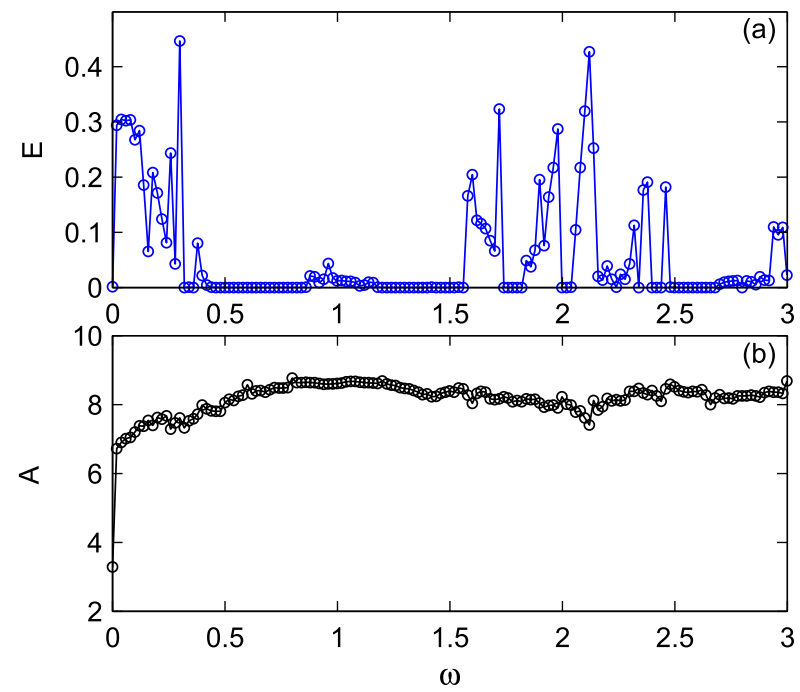

Figure 2. (a) Normalized average synchronization error $E$ and (b) average amplitude $A$ for a dynamic conjugate coupling between two Rössler oscillators as a function of switching frequency $\omega$ for $k_{1}=0.06$ and $k_{2}=0.196$.

$\omega$ for the Rössler oscillators with dynamic conjugate coupling for $\mathrm{C} 2$ in figure $3 \mathrm{a}-\mathrm{b}$. We observed three different regions of oscillations, which shows the revival of oscillations in the system. The first window is in the range of $\omega=1.39$ to 1.53 , while the other windows are observed in the range of $\omega$ from 1.66 to 1.83 and $\omega=2.08$ to 2.29 (see figure $3 b$ ). In all these windows, we observed the intermittent state (IMS) having intermittency between the oscillation and amplitude death state. To study the intermittent state, we have also introduced another order parameter $A_{\text {ims }}$ based on calculating the amplitude of coupled systems in a finite time interval and it is defined ${ }^{\dagger}$. If $0<A_{\text {ims }}<1$, we observed the intermittent state in coupled systems. The time series of $x$ variables for $\omega=2.2$ is plotted in figure 3c which shows the system dynamics switching between three dynamical states: complete synchronization, anti-phase synchronization and amplitude death states. Here, inset figures show that initially the system dynamics is in an anti-phase synchronization state but after the death state the system is in complete synchronization state. To explore the dynamical regimes, in this case, we draw a parameter plane in $\omega-k_{2}$ at fixed value of $k_{1}=0.15$ in figure $3 \mathrm{~d}$, where NS, CS, IMS and AD regimes are observed. All these numerical results have been

\footnotetext{
${ }^{\dagger} A_{\text {ims }}$ is fraction of total oscillatory states in finite time interval to total time interval and it is defined by $A_{i m s}=\frac{1}{n} \sum_{i=1}^{n} R_{i}$, where $R_{i}=1$ if $A>0$ for time interval [ $(i-1) \Delta t, i \Delta t]$, otherwise $R_{i}=0$. Total number of time interval $n=T / \Delta t$, where $T$ is total time and $\Delta t$ is time interval. In numerical calculation, we have considered the $\Delta t=10^{3}$
} 

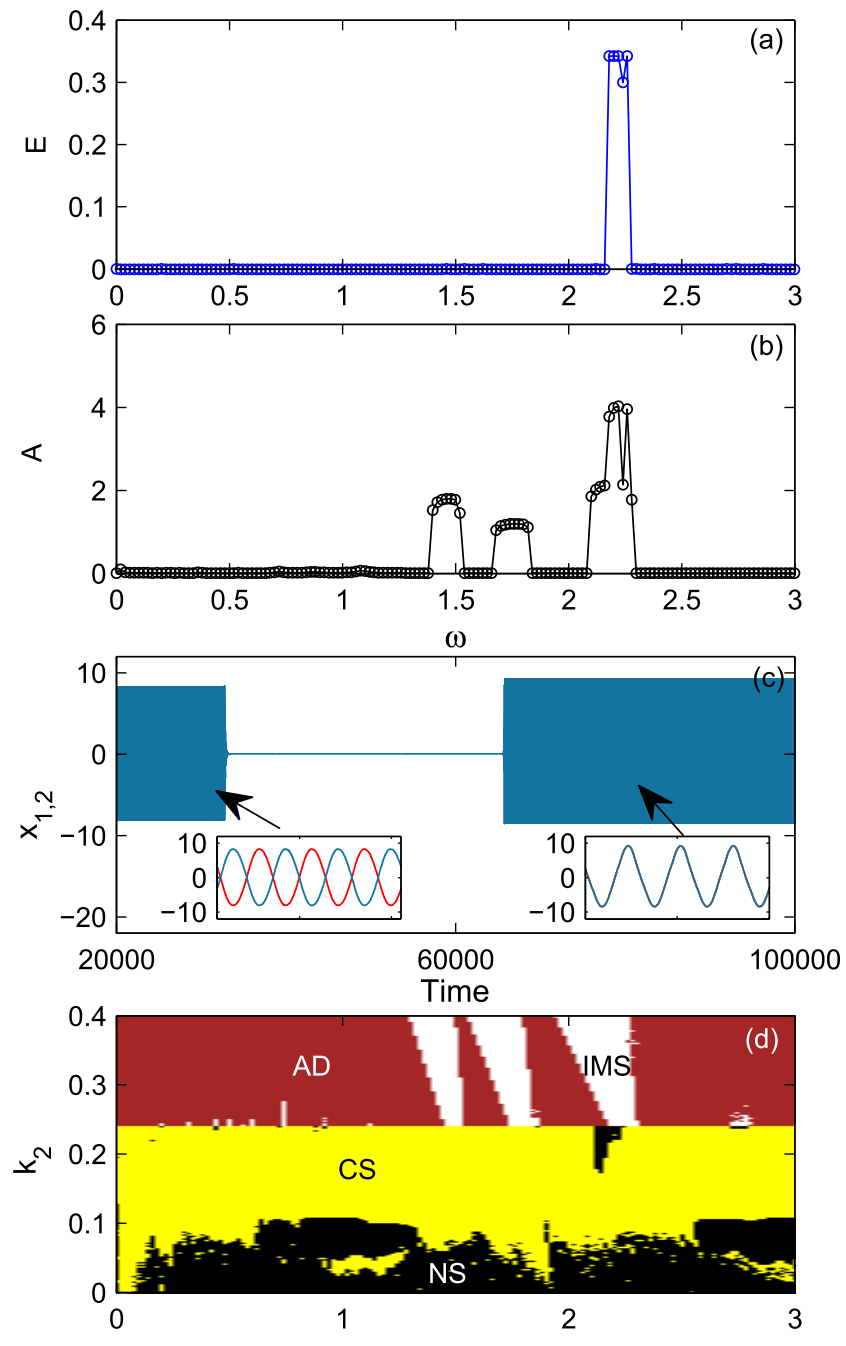

$\omega$

Figure 3. (a) Normalized average synchronization error $E$ and (b) average amplitude $A$ as a function of switching frequency $\omega$ for $k_{1}=0.15$ and $k_{2}=0.3$ for $\mathbf{C 2}$ case. (c) The time series of $x$ variables for $\omega=2.2$. Inset figures show anti-phase and in-phase synchronization respectively. (d) Different dynamical regimes of the coupled Rössler oscillators with dynamic conjugate coupling in parameter space $k_{2}-\omega$ for fixed value of $k_{1}=0.15$. The regions having states namely, no-synchronization (NS), complete synchronization (CS), intermittent state (IMS), and amplitude death (AD) respectively are shown.

computed for a time of $10^{5}$ after removing transients for $10^{4}$ units of time.

Next, we consider the Truscott-Brindley (TB) model which was introduced to model the dynamics of phytoplankton and zooplankton in the ocean [22]. The model imitates the emergence of a plankton bloom using the concept of an excitable system. The dynamical equations of time-varying conjugate interaction in such a model system are as follows, $\dot{x}_{i}=r x_{i}\left(1-x_{i}\right)-y_{i}\left(\frac{x_{i}^{2}}{v^{2}+x_{i}^{2}}\right)+k(t)\left(y_{j}-x_{i}\right)$

$\dot{y}_{i}=a\left(\frac{x_{i}^{2}}{v^{2}+x_{i}^{2}}-s\right) y_{i}$

where $x$ and $y$ represent the prey and predator populations respectively. The growth rate of the prey is represented by a logistic growth function, with the maximum growth rate $r$. Predation by the predator is represented by a Holling type III function, $v$ is the half saturation constant for the predator which governs how quickly the maximum predation rate is achieved as the prey population increases. The constant $a$ models the maximum growth rate of the predator. Here, we have assumed that there is no inter-species competition of the two prey species; each of them can grow to its own carrying capacity in the absence of predation. Both prey species are consumed by the predator following cross-predation terms with factor $k(t)$ which depends on switching frequency $\omega$. The parameters of the system are $r=0.43$, $v=0.053, s=0.56$ and $a=0.05$. In the case of static coupling, for $k=0.02$, the prey population of one of the systems reduces drastically. Further increasing $k$ leads
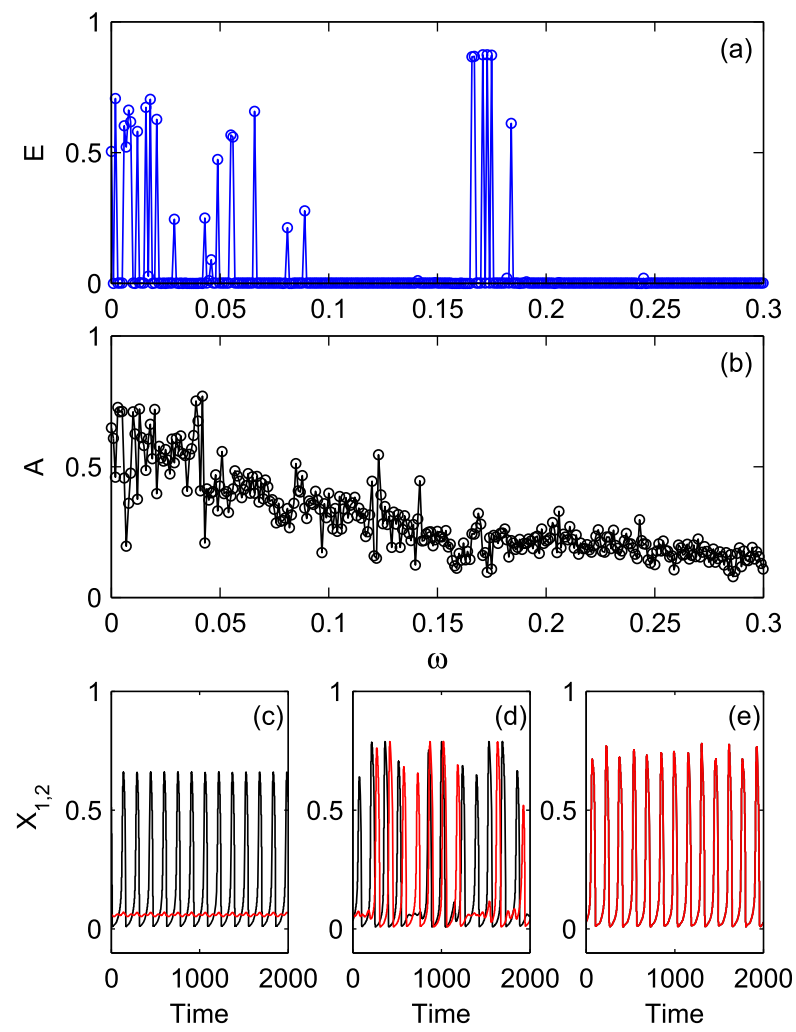

Figure 4. (a) Normalized average synchronization error $E$ and (b) average amplitude $A$ as a function of switching frequency for the prey-predator model with conjugate coupling. Time series of prey population of the coupled system at (c) $\omega=0$, (d) $\omega=0.01$, and (e) $\omega=0.1$ respectively. 
the system dynamics to oscillation death (results are not shown here).

Now, we consider switching coupling in the system by introducing $k(t)$ as the time-varying coupling strength. $k(t)$ is the same as defined above and switches between the two coupling strengths $k_{1}$ and $k_{2}$. We choose $k_{1}=0$ and $k_{2}=0.02$. Both of these cases exclude the possibility of synchronization when $k_{1}=k_{2}$. The numerical calculations in this case are computed for $10^{4}$ time units, after removing transients of $10^{4}$ time units. Results for the switching interaction in conjugate coupling are shown in figure 4 . Normalized average synchronization error $E$ is plotted as a function of switching frequency in figure $4 \mathrm{a}$. At lower switching frequency $\omega<0.1$, we observed the mixed state of synchronization and unsynchronized motion in periodic and aperiodic states in coupled prey-predator model. While at higher values of $\omega>0.1$, the coupled system settles down to the synchronization state in aperiodic state. We have also observed the unsynchronized dynamics in a small range between 0.16 to 0.18 for higher values of switching frequency $\omega$. The average amplitude of coupled prey-predator model is shown in figure $4 \mathrm{~b}$, which shows that the amplitude of oscillations decreases with switching frequency. The time series of coupled prey-predator model are shown in figure 4c-e at $\omega=0,0.01$ and 0.1 respectively. It is also evident from these that the amplitude of one of the oscillators has increased considerably by increasing the switching frequency $\omega$. It also shows that the prey and predator population in a system can grow if the strength of cross-predation changes with time rather than being constant in time.

\section{Conclusion}

The main concern of this paper is to examine the dynamics of time-varying interaction in nonlinear oscillators coupled through conjugate variables, where the coupling strength switches periodically between two constant values. To demonstrate such a time-varying coupling, we have studied two dynamical systems, Rössler oscillator, and prey-predator model.

We have studied different cases depending on the choice of coupling strength in the different dynamical regimes between which it switches. We have observed revival of oscillation in the coupled Rössler systems with an intermittent state, where the dynamics switches between different dynamical states, like synchronized state and amplitude death state with time-varying conjugate coupling. These dynamical behaviors depend on the switching frequency of coupling strength. We have also demonstrated the time-varying conjugate coupling in a prey-predator population model. The present results are of potential utility in appropriate design strategies and/or understanding of complex systems with dynamic interaction leading to synchronized, intermittent, and oscillation suppression states.

\section{Acknowledgements}

MDS acknowledges the financial support from DST, New Delhi.

\section{References}

[1] L Glass, Nature 410, 277 (2001)

[2] A Pikovsky, M Rosenblum and J Kurths, Synchronization: A Universal Concept in Nonlinear Sciences (Cambridge university press, 2003)

[3] D V R Reddy, A Sen and G L Johnston, Phys. Rev. Lett. 80, 5109 (1998)

[4] R Karnatak, R Ramaswamy and A Prasad, Phys. Rev. E 76, 035201 (2007)

[5] A Prasad, M Dhamala, B M Adhikari and R Ramaswamy, Phys. Rev. E 81, 027201 (2010)

[6] R Karnatak, R Ramaswamy and A Prasad, Chaos 19, 033143 (2009)

[7] A Sharma, M D Shrimali, A Prasad, R Ramaswamy and U Feudel, Phys. Rev. E 84, 016226 (2011)

[8] M Kim, R Roy, J L Aron, T W Carr and I B Schwartz, Phys. Rev. Lett. 94, 088101 (2005)

[9] R Karnatak, R Ramaswamy and U Feudel, Chaos Solitons \& Fractals 68, 48 (2014)

[10] M Dasgupta, M Rivera and P Parmananda, Chaos 20, 023126 (2010)

[11] T Singla, N Pawar and P Parmananda, Phys. Rev. E 83, 026210 (2011)

[12] L Song, M Kolar and E P Xing, Bioinformatics 25, i128 (2009)

[13] I V Belykh, V N Belykh and M Hasler, Physica D 195, 188 (2004)

[14] L O Chua, T Yang, G Q Zhong and C W Wu, IEEE Trans. Circuits Syst. I Fundam. Theory Appl. 43, 862 (1996)

[15] A Prasad, Pramana J. Phys. 81, 407 (2012)

[16] V Kohar, P Ji, A Choudhary, S Sinha and J Kurths, Phys. Rev. E 90, 022812 (2014)

[17] S S Chaurasia, A Choudhary, M D Shrimali and S Sinha, arXiv: 1701.07314v1 (2017)

[18] V Kohar and S Sinha, Chaos, Solitons Fractals 54, 127 (2013)

[19] P So, B C Cotton and E Barreto, Chaos 18, 037114 (2008)

[20] A Buscarino, M Frasca, M Branciforte, L Fortuna and J C Sprott, Nonlinear Dynamics 88, 673 (2017)

[21] A Sharma, M D Shrimali and K Aihara, Phys. Rev. E 90, 062907 (2014)

[22] J Truscott and J Brindley, Bull Math Biol 56, 981 (1994) 